\title{
UNIFORM EQUIVALENCE BETWEEN BANACH SPACES
}

\author{
BY ISRAEL AHARONI AND JORAM LINDENSTRAUSS
}

Communicated by P. T. Church, August 5, 1977

It is a well-known result of Kadec that every two separable infinite dimensional Banach spaces are homeomorphic. Also in large classes of nonseparable Banach spaces (perhaps all) the density character of a Banach space is its only topological invariant (see the book [2] for details). The situation changes considerably if we consider uniform homeomorphisms. Several results are known which prove the nonexistence of uniform homeomorphisms between certain Banach spaces of the same density character. As a matter of fact, the following problem was raised by many mathematicians: Do there exist two nonisomorphic Banach spaces which are uniformly homeomorphic? (i.e. does the uniform structure of a Banach space determine its linear structure?) For a recent survey of results related to this problem see [3].

While studying the question of existence of nonlinear liftings, we found in a surprisingly simple manner an example which answers this problem. Let $\Gamma$ be a set of the cardinality of the continuum. Then $c_{0}(\Gamma)$ is Lipschitz equivalent to a certain closed subspace $X$ of $l_{\infty}$ (i.e. there is a map $T$ from $c_{0}(\Gamma)$ onto $X$ so that $T$ and $T^{-1}$ satisfy a Lipschitz condition). Since there is no sequence of continuous linear functionals which separate the points in $c_{0}(\Gamma)$, this space is not isomorphic to a subspace of $l_{\infty}$.

Let $U \supset V$ be Banach spaces and let $\varphi: U \rightarrow U / V$ be the quotient map. We say that $\varphi$ admits a Lipschitz (resp. uniformly continuous) lifting if there is a Lipschitz (resp. uniformly continuous) map $\psi: U / V \rightarrow U$ so that $\varphi \psi$ is the identity of $U / V$. If such a lifting exists then $U$ is Lipschitz (respectively uniformly) homeomorphic to the direct sum $V \oplus U / V$. A suitable map $T$ which establishes the homeomorphism is $T u=(u-\psi \varphi(u), \varphi(u))$.

Let $\left\{N_{\gamma}\right\}_{\gamma \in \Gamma}$ be a collection of subsets of the integers $N$ so that each $N_{\gamma}$ is infinite and $N_{\gamma} \cap N_{\gamma^{\prime}}$ is finite for $\gamma \neq \gamma^{\prime}$. Let $X$ be the closed linear subspace of $l_{\infty}$ spanned by $c_{0}$ and the characteristic functions $\chi_{\gamma}$ of $N_{\gamma}, \gamma \in \Gamma$. Clearly $X / c_{0}$ is isometric to $c_{0}(\Gamma)$ with $\varphi \chi_{\gamma}$ corresponding to the natural unit vectors $e_{\gamma}$ of $c_{0}(\Gamma)$. The map $\varphi$ admits a Lipschitz lifting and thus $X$ is Lipschitz equivalent of $c_{0}(\Gamma) \oplus c_{0} \approx c_{0}(\Gamma)$. Indeed, let

$$
y=\sum_{n=1}^{t} a_{n} e_{\gamma_{n}}-\sum_{m=1}^{s} b_{m} e_{\gamma_{m}^{\prime}}
$$

AMS (MOS) subject classifications (1970). Primary 57A17; Secondary 54E15. 
with $a_{i} \geqslant a_{2} \geqslant a_{3}, \ldots, b_{1} \geqslant b_{2} \geqslant b_{3}, \ldots, 0 \leqslant s, t \leqslant \infty$, be the unique representation of $y \in c_{0}(\Gamma)$ as a difference of disjointly supported positive elements $y^{+}$and $y^{-}$(we assume also that $\gamma_{i} \neq \gamma_{j}, \gamma_{i}^{\prime} \neq \gamma_{j}^{\prime}$ for $i \neq j$ ). Put

$$
\begin{aligned}
M_{n} & =N_{\gamma_{n}} \sim\left(\bigcup_{i=1}^{n-1} N_{\gamma_{i}}\right), M_{m}^{\prime}=N_{\gamma_{m}^{\prime}} \sim\left(\bigcup_{j=1}^{m-1} N_{\gamma^{\prime}}\right) \\
\psi(y) & =\sum_{n=1}^{t} a_{n} \chi_{M_{n}}-\sum_{m=1}^{s} b_{m} \chi_{M^{\prime}{ }_{m}}
\end{aligned}
$$

where $\chi_{A}$ denotes the characteristic function of a set $A$. It is evident that $\varphi \psi=$ identity of $c_{0}(\Gamma)$. That $\psi$ is a Lipschitz map, follows from the following easily verified formula. For $y \in c_{0}(\Gamma), n \in N$ we have

$$
\psi(y)(n)=\operatorname{dist}\left(y^{+}, Z_{n}\right)-\operatorname{dist}\left(y^{-}, Z_{n}\right)
$$

where

$$
Z_{n}=\operatorname{span}\left\{e_{\gamma} ; n \notin N_{\gamma}\right\} .
$$

REMARKS. (i) It is of course of interest to find also separable examples. Plausible separable candidates are the pairs $c_{0}$ and $c\left(\omega^{\omega}\right)$ or even $c_{0}$ and $C(0,1)$ (cf. [1]).

(ii) In the linear case the existence of a bounded lifting to the quotient map $U \rightarrow U / V$ is of course equivalent to the existence of a bounded projection from $U$ onto $V$. In the nonlinear case the existence of a lifting trivially implies the existence of a projection, but the converse is not true in general. For example let $D$ be the space of all real-valued functions on $[0,1]$ such that $\lim _{h \downarrow 0} f(t-h)$ exists and $f(t)=\lim _{h \downarrow 0} f(t+h)$ for every $t$ and so that the only possible jumps of $f$ are for rational $t$, with

$$
\|f\|=\sup \{|f(t)|, 0 \leqslant t \leqslant 1\} .
$$

Then $D \supset C(0,1)$, there is a Lipschitz projection from $D$ onto $C(0,1)$ (by [4]) but there is no Lipschitz lifting $\psi$ from $c_{0}(Q)=c_{0}=D / C(0,1)$ into $D$ (where $Q$ denotes the rationals). Indeed suppose that such a $\psi$ exists and let $K$ be its Lipschitz constant. Let $x, y \in c_{0}(Q)$ be such that $\|\psi(x)-\psi(y)\|>(K-1 / 4)$ $\|x-y\|$. Then there is an open set $G$ in $[0,1]$ so that

$$
|\psi(x)(t)-\psi(y)(t)|>(K-1 / 4)\|x-y\|
$$

for $t \in G$ and so that both $\psi(x)$ and $\psi(y)$ vary by less than $\|x-y\| / 8$ on $G$. Let $q$ be a rational point in $G$ so that the unit vector $e_{q}$ in $c_{0}(Q)$ satisfies

$$
\|x-y \pm\| x-y\left\|e_{q} / 2\right\|=\|x-y\|,
$$

and put $z=(x+y) / 2+\|x-y\| e_{q} / 4$. Then $\|z-x\|=\|z-y\|=\|x-y\| / 2$ and hence 


$$
\|\psi(z)-\psi(x)\|,\|\psi(z)-\psi(y)\| \leqslant K\|x-y\| / 2 .
$$

In particular we get that $|\psi(z)(t)-(\psi(x)(t)+\psi(y)(t)) / 2|<\|x-y\| / 8$ for $t \in G$ but this contradicts the fact that $\psi(z)$ has a jump of size $\|x-y\| / 2$ at $q$. A slight variant of this argument shows that $\varphi: D \rightarrow D / C(0,1)$ does not admit even a uniformly continuous lifting.

(iii) It follows from results of [4] that in many cases (e.g. if $V$ is reflexive) the existence of a uniformly continuous lifting to $\varphi: U \rightarrow U / V$ already implies the existence of a bounded linear lifting (i.e. that $V$ is complemented in $U$ ).

\section{REFERENCES}

1. I. Aharoni, Every separable metric space is Lipschitz equivalent to a subset of $c_{0}$, Israel J. Math. 19 (1974), 284-291.

2. C. Bessaga and A. Pelczyński, selected topics in infinite-dimensional topology, Monografie Mat., No. 58, Warsaw, 1975.

3. P. Enflo, Uniform homeomorphisms between Banach spaces, Seminaire MaureySchwartz 1975-1976, Expose \#18.

4. J. Lindenstrauss, On nonlinear projections in Banach spaces, Michigan Math. J. 11 (1964), 263-287.

INSTITUTE FOR ADVANCED STUDIES, THE HEBREW UNIVERSITY, JERUSALEM 